\title{
Direct laboratory observation of patchy saturation and its effects on ultrasonic velocities
}

\author{
Maxim Lebedev and Jullanna Toms-Stewart, Curtin University \\ Ben Clennell, Marina Pervukhina, Valeriya Shulakova, Lincoln Paterson and Tobias M. Müller, CSiro Petroleum \\ BORIS GUREVICH, Curtin University and CSIRO Petroleum \\ FABIAN WenZLAU, Karlsruhe University
}

$\mathrm{M}$ aximizing the recovery of known hydrocarbon reserves is one of the biggest challenges facing the petroleum industry today. Optimal production strategies require accurate monitoring of production-induced changes of reservoir saturation and pressure over the life of the field. Time-lapse seismic technology is increasingly used to map these changes in space and time. However, until now, interpretation of time-lapse seismic data has been mostly qualitative. In order to allow accurate estimation of the saturation, it is necessary to know the quantitative relationship between fluid saturation and seismic characteristics (elastic moduli, velocity dispersion, and attenuation). The problem of calculating acoustic properties of rocks saturated with a mixture of two fluids has attracted considerable interest (Gist, 1994; Mavko and Nolen-Hoeksema, 1994; Knight et al., 1998. For a comprehensive review of theoretical and experimental studies of the patchy saturation problem see Toms et al., 2006).

For a porous rock whose matrix is elastically homogeneous and inhomogeneities caused only by spatial variations in fluid properties, two theoretical bounds for the P-velocity are known (Mavko and Mukerji, 1998; Mavko et al., 1998). In the static (or low-frequency) limit, saturation can be considered homogeneous, and, hence, the rock may be looked at as saturated with a homogeneous mixture of the fluids. In this case, the bulk modulus of the rock is defined by the Gassmann equation with the fluid bulk modulus given by Wood's formula, i.e., the saturation-weighted harmonic average of the bulk moduli of fluids. The Gassmann-Wood bound is valid when the characteristic patch size is small compared to the fluid diffusion length. The diffusion length is primarily controlled by rock permeability, fluid viscosity, and wave frequency. In the opposite case, when the patch size is much larger than the diffusion length, there is no pressure communication between fluid pockets, and, consequently, no fluid flow occurs. In this no-flow (or high-frequency) limit, the overall rock behaves like an elastic composite consisting of homogeneous patches whose elastic moduli are given by Gassmann's theory. Since all these patches have the same shear modulus, the effective Pwave modulus can be obtained using Hill's equation, i.e, the saturation-weighted harmonic average of the P-wave moduli. The Gassmann-Wood and Gassmann-Hill bounds apply in the low- and high-frequency limits, respectively. For intermediate frequencies, uneven deformation of fluid patches by the passing wave results in local pressure gradients and hence, in the wave-induced fluid flow, which in turn causes wave attenuation and velocity dispersion. For regular distributions of fluid patches of simple geometry (spheres, flat slabs), these effects have been first studied by White (1975), White et al.
(1975), and Dutta and Ode (1979). More recently, the effect of regularly distributed patches of more general shape was modeled by Johnson (2001) and Pride et al. (2004). For randomly distributed fluid patches Müller and Gurevich (2004) and Müller et al. (2008) showed how the effect of wave-induced flow controls the transition from the Gassmann-Wood to the Gassmann-Hill bounds.

While theoretical poroelastic models can predict the acoustic response for a given spatial distribution of fluid patches, the factors controlling the formation of the patches are less understood. These factors can be studied using fluidinjection experiments in the laboratory. Previously reported laboratory observations demonstrate a qualitative link between fluid patch distribution and acoustic velocities (Cadoret et al., 1995, 1998; Monsen and Johnstad, 2005). In order to get a deeper insight into the factors influencing the patch distribution and the associated wave response, we perform simultaneous measurements of $\mathrm{P}$-wave velocities and rock sample X-ray computer tomography (CT) imaging. The CT imaging allows us to infer the fluid distribution inside the rock sample during saturation (water imbibition). We then show that the experimental results are consistent with theoretical predictions and numerical simulations.

\section{Experimental setup}

Experiments are performed on a cylindrical sample $(38 \mathrm{~mm}$ in diameter and $60 \mathrm{~mm}$ long) cut from a Casino sandstone (Otway Basin, Australia). The sample is dried at $100^{\circ} \mathrm{C}$ under reduced pressure for 24 hours. The petrophysical properties are measured using a Coretest AP-608 automatic permeameter/porosimeter (Table 1). Then, the sample is sealed with a thin epoxy layer in order to prevent fluid leakage through the surface. Longitudinal $\left(V_{p}\right)$ and shear-wave $\left(V_{s}\right)$ velocities at $1 \mathrm{MHz}$ are measured in the direction across to the core axis (perpendicular to the fluid flow) using broadband ultrasonic transducers. Intermediate aluminum "guide pins" are placed between the sample and transducers to secure sufficient and constant coupling, as well as to provide transparency for Xray radiation.

\begin{tabular}{|l|c|}
\hline & Casino Otway \\
\hline Bulk density, $\mathrm{g} / \mathrm{cm}^{3}$ & 2.2 \\
\hline Grain density, $\mathrm{g} / \mathrm{cm}^{3}$ & 2.65 \\
\hline Porosity $\%$ & 16.7 \\
\hline Permeability, $\mathrm{mD}$ & 7.26 \\
\hline
\end{tabular}

Table 1: Petrophysical properties of the dry rock sample. 


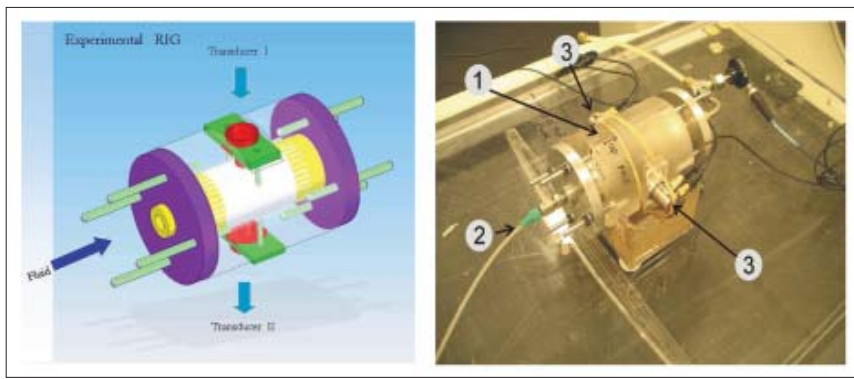

Figure 1. Core sample jacketed inside experimental rig: 1-X-ray transparent jacket; 2-injection pipe; 3-ultrasonic transdusers.

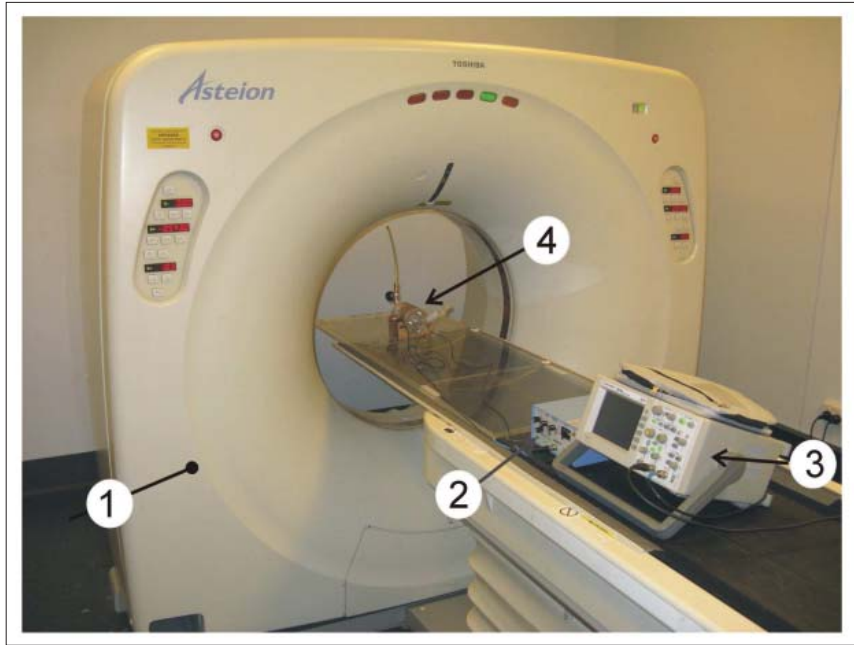

Figure 2. Simultaneous acquisition of acoustic properties and fluid saturation using ultrasonic transducers and CT scan: 1-computer tomograph; 2-ultrasonic pulse receiver; 3-oscilloscope; 4-jacketed sample.

Two different saturation methods-referred to as dynamic and quasi-static saturation-are used in this study. In the dynamic saturation experiments, the samples are jacketed in the experimental cell made out of X-ray transparent material PMMA (Figure 1). Distilled water is injected into the sample from one side. The injection rate is $10 \mathrm{ml}$ per day. In quasi-static saturation experiments, the samples are saturated during a long period of time (up to two weeks) under reduced pressure in order to achieve near-uniform fluid distribution for a given saturation level. For quasi-static experiments, the saturation level is determined by measurement of the volume (weight) of water fraction divided by the total volume of pores. A less than $1 \%$ difference in velocities measured in different directions indicates that fluid is uniformly distributed within the sample.

The fluid distribution in the dynamic saturation experiments (both spatial and time dependence) is characterized using X-ray computer tomography. (Figure 2). The resolution is $0.2 \times 0.2 \times 1 \mathrm{~mm}^{3}$ (voxel size). This resolution is not sufficient to image the exact fluid patch geometry; however, it shows the overall character of the fluid distribution. The fluid saturation is estimated as a difference in average CT number (a value related to material density) between the saturated and dried sample divided by the volume of porous fraction. The temporal evolution of the saturation profile along the rock

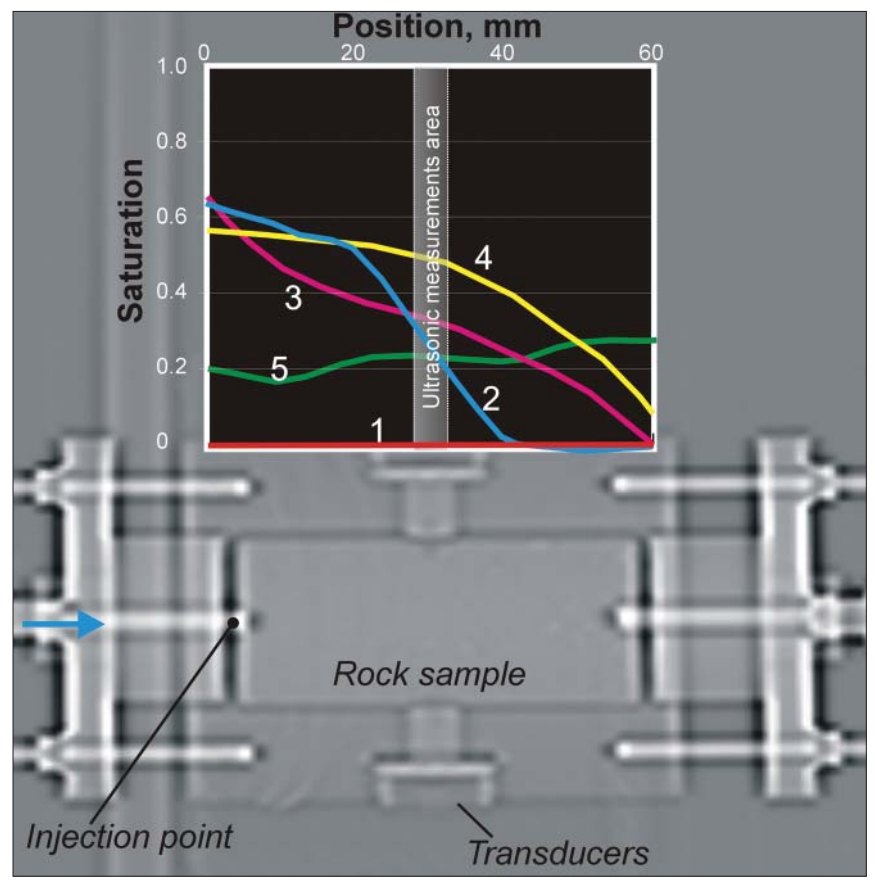

Figure 3. X-ray image of the whole core sample. The embedded plot shows the profiles of water saturation along the $60 \mathrm{~mm}$ sample at different times (increasing from 1 to 5) during the saturation experiment.

sample is shown in Figure 3. We see that the overall saturation profile becomes progressively more uniform over time. All experiments are performed in a laboratory environment at $25^{\circ} \mathrm{C}$.

\section{Experimental results}

Velocity is obtained by first-break picking on output signal. Figure 4 shows the P-wave velocity as a function of saturation for the Casino Otway sandstone from the dynamic saturation experiment (empty symbols) and quasi-static saturation experiment (solid symbols). A transition from the GassmannWood to Gassmann-Hill bound is clearly observed for water saturations of $40-50 \%$ for dynamic saturation and $60-70 \%$ for quasi-static saturation. Figure 5 shows CT images recorded during the dynamic saturation experiments. Scans 1-5 correspond to points $1-5$ on the velocity-saturation curve (Figure 4): increasing saturation from the dried sample (1) up to $50 \%$ (point 4 ) and then decreasing saturation down to $26 \%$ (point 5). At low saturations, the velocity can be well described by the Gassmann-Wood relation; however, a sharp increase in velocity is observed when saturation exceeds $40 \%$ (points 3 and 4 in Figure 4). Then, the velocity-saturation approaches the Gassmann-Hill bound.

We also observe that for relatively large fluid injection rates (dynamic experiment) the transition from homogeneous to patchy saturation (from the Gassmann-Wood to GassmannHill) occurs at smaller degrees of saturation as compared with low injection rates (quasi-static experiment). This observation suggests that the process of fluid patch formation is controlled by the injection rate. 

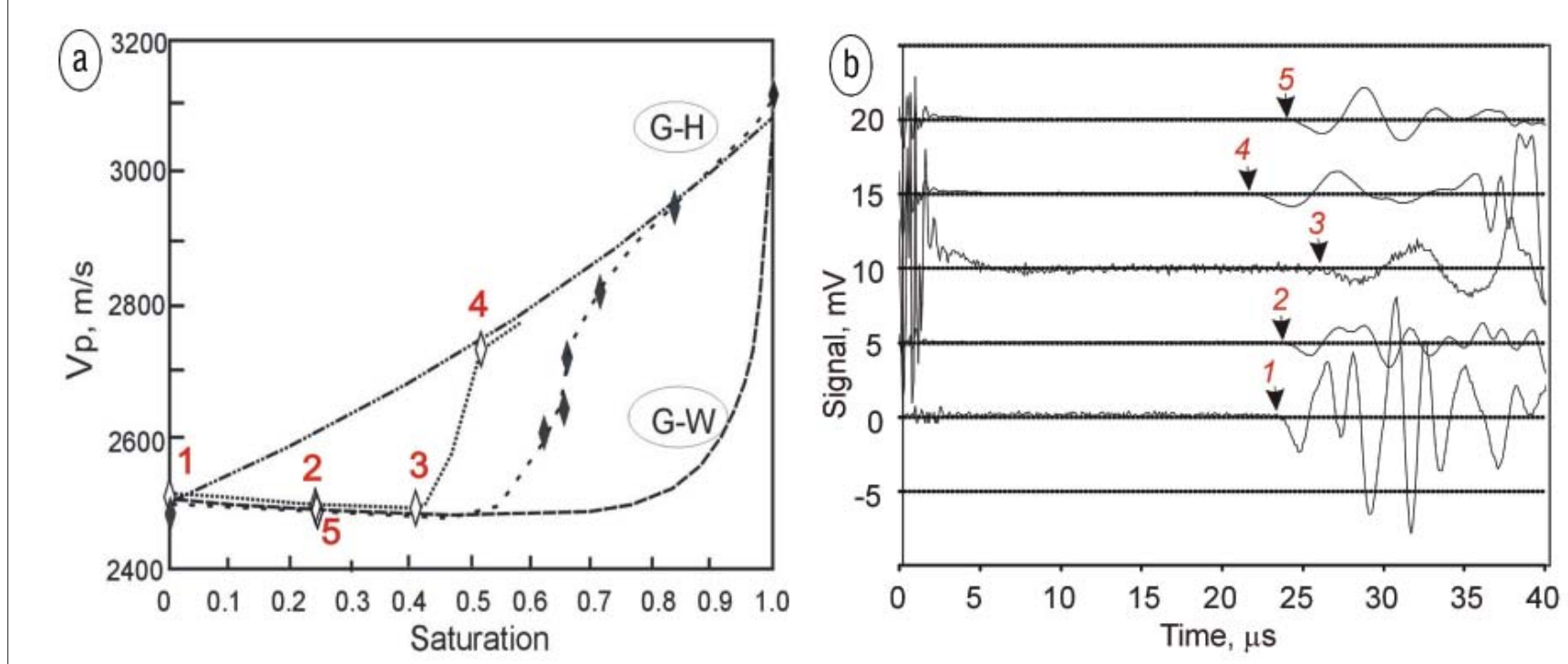

Figure 4. (a) Velocity versus saturation for the Casino Otway Basin sandstone from the quasi-static saturation experiment (black diamonds) and dynamic saturation experiment (white diamonds). The numbers from 1 to 5 indicate acoustic measurements corresponding to the CT images shown in Figure 5, and saturation profiles shown in Figure 3. Theoretical Gassmann-Wood (G-W) and Gassmann-Hill (G-H) bounds are shown by dashed and dash-dotted lines, respectively. (b) Output signals corresponding to five stages during the dynamic fluid-injection experiment.

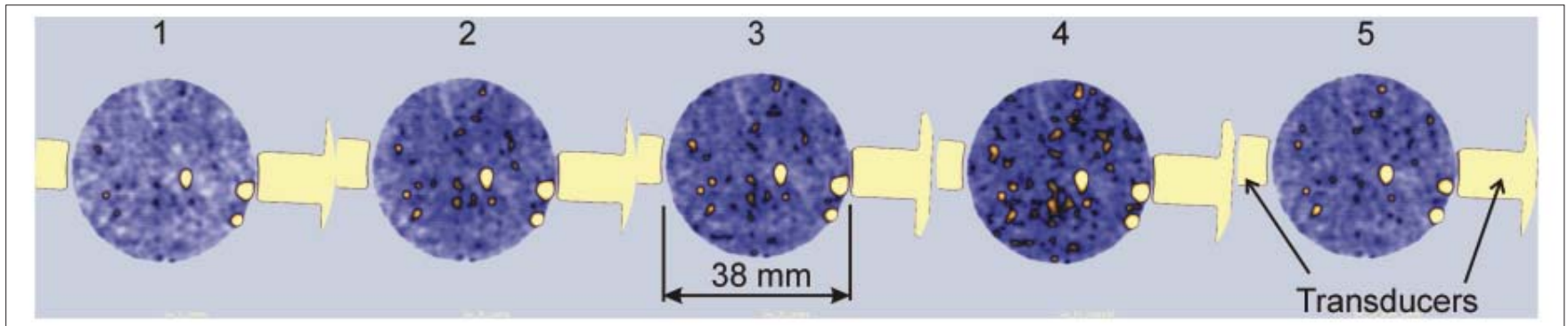

Figure 5. CT images of the Casino Otway Basin sandstone at different times after start of injection at the same position: 1-dried sample; 2-1 h; 3-24 h; 4-72 h; 5-after gas injection. Contours of guide pins are visible at right and left side of the sample.

\section{Discussion}

The velocity-saturation relation shows a transition from the Gassmann-Wood to the Gassmann-Hill behavior. Characteristics of this transition can be related to the parameters controlling the wave-induced flow of the pore fluids in a poroelastic solid. There are two important length scales involved. One is the diffusion length (the wavelength of Biot's slow wave) $\lambda_{D}$, which is controlled by properties of the rock matrix, the pore fluids, and signal frequency; in our experiment it is on the order $\lambda_{D} \approx 0.1 \mathrm{~mm}$. The other important length scale is the characteristic size of the fluid patches, $a$. As mentioned earlier, the velocity value between the GassmannWood and Gassmann-Hill bound is controlled by the ratio of these two length parameters. However it is important to note that the patch size is likely to vary with the degree of saturation, and that this variation may itself be rate-dependent. The CT scans in Figure 5 illustrate that for saturations of more than $40 \%$, the fluid patches begin to cluster, effectively forming larger patches spanning a few millimeters in diameter (scan 4). Then, $\lambda_{D}<<a$, and hence, the proximity to the Gassmann-Hill bound (as shown in Figure 4a) is expected. Conversely, for small saturations, the condition $\lambda_{D}>>a$ seems to apply, and, therefore, the velocities follow the Gassmann-Wood prediction. The transition between these two bounds occurs if $\lambda_{D} \approx a$. Then, the mechanism of wave-induced flow takes place resulting in dispersion and attenuation of the ultrasonic signals. That is to say, the transition behavior in the velocity-saturation plane is controlled by the interplay between Biot's slow wave created at fluid-gas interfaces and the characteristic size/distribution of the fluid patches. This interpretation is consistent with recently proposed theoretical models, which take into account the randomness of the patch distribution (Toms et al., 2007).

In order to validate our interpretation of the velocitysaturation relation in terms of wave-induced flow, we also perform numerical simulations of wave propagation in a $2 \mathrm{D}$ poroelastic solid. The simulations are performed using 2D finite-difference solver of Biot's equations of dynamic poroelasticity (Krzikalla and Müller, 2007). Figure 6 shows the modeled velocity-saturation relation obtained for material constants and wave frequency used in the laboratory experiment described above. The degree of saturation is varied by 


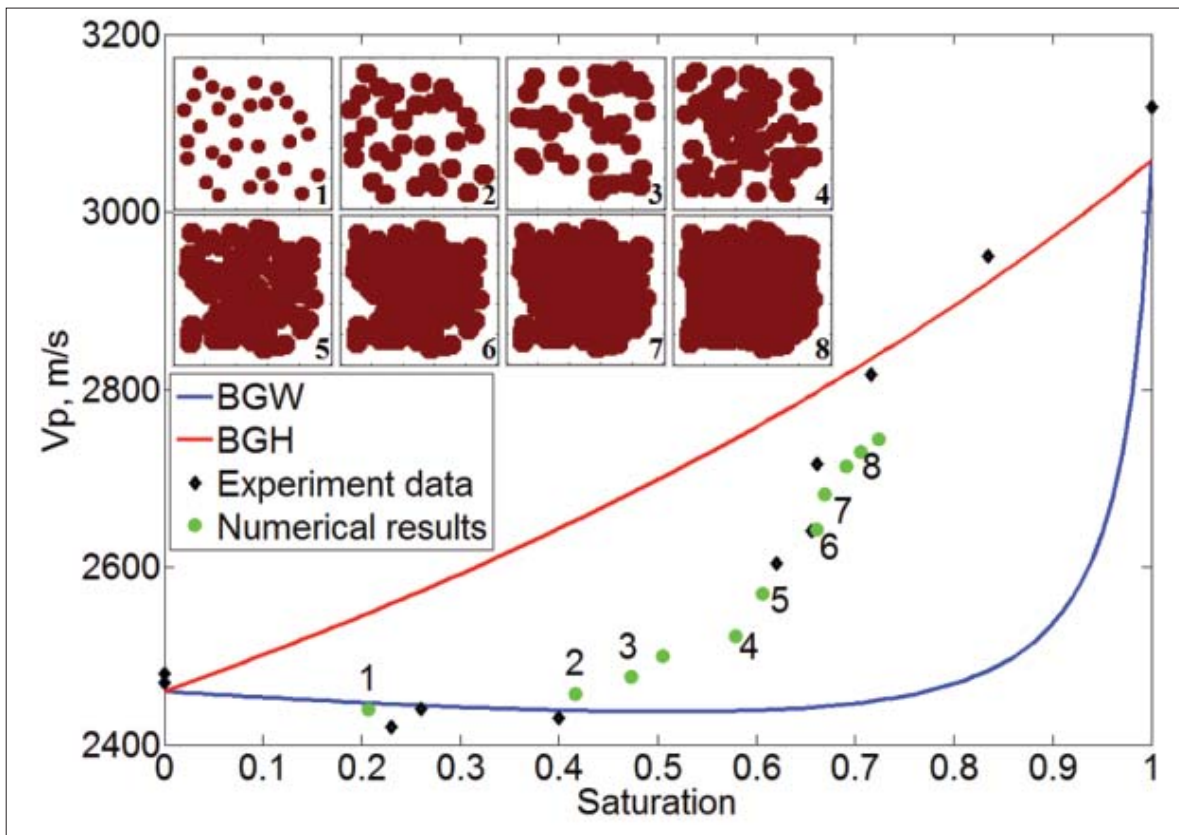

Figure 6. Velocity-saturation relation determined from numerical simulations of wave propagation in a poroelastic solid with randomly distributed patches that cluster for larger saturation values (see inset). Experimentally determined velocities for the quasi-static injection experiment are also shown.

increasing the size of the randomly distributed fluid patches. It can be seen that for large saturations, the fluid patches begin to cluster and resemble the clustering observed in the CT scans. Despite the simplified numerical setup (2D modeling only and patch distribution not derived directly from the CT scans), the simulation results reproduce the overall behavior of the measured velocity-saturation relation. This confirms that the transition observed in the ultrasonic measurements can be attributed to the mechanism of wave-induced flow. We plan to extend our numerical simulations to $3 \mathrm{D}$ so that the $3 \mathrm{D}$ patch distributions reconstructed from CT images can be directly used as input model in our simulations.

In this work, no attempt has been made to characterize the fluid patch distribution in a quantitative way from the CT scans; this requires further research. Recently, Toms et al. (2007c) constructed gas saturation maps from X-ray CT scans and analyzed their statistical properties. Though the CT image resolution is too low in order to reveal individual fluid patches, characteristic length scales of the patch size and distribution can be inferred from the spatial correlation function and the chord length density function. These statistical measures can then be used together with the theoretical models of random patchy saturation developed by Toms et al. (2007a, b) and Müller et al. (2008).

Suggested reading. "Influence of frequency and fluid distribution on elastic wave velocities in partially saturated limestones" by Cadoret et al. (Journal of Geophysical Research, 1995). "Fluid distribution effect on sonic attenuation in partially saturated limestone" by Cadoret et al. (Geophysics, 1998). "Attenuation and dispersion of compressional waves in fluid-filled porous rocks with partial gas saturation-White model-Part II:
Results" by Dutta and Ode (GeophysICS, 1979). "Interpreting laboratory velocity measurements in partially gassaturated rocks" by Gist (GeophysICS, 1994). "Theory of frequency dependent acoustics in patchy-saturated porous media" by Johnson (Journal of the Acoustical Society of America, 2001). "Acoustic signatures of partial saturation" by Knight et al. (Geophysics, 1998). "High-contrast finite-differences modeling in heterogeneous poroelastic media" by Krzikalla and Müller, (SEG 2007 Expanded Abstracts). "Bounds on low-frequency seismic velocities in partially saturated rocks" by Mavko and Mukerji (Geophysics, 1998). "Estimating seismic velocities at ultrasonic frequencies in partially saturated rocks" by Mavko and Nolen-Hoeksema (GeoPHYsics, 1994). The Rock Physics Handbook: Tools for Seismic Analysis in Porous Media by Mavko et al. (Cambridge, 1998). "Improved understanding of velocity-saturation relationships using 4D computer-tomography acoustic measurements" by Monsen and Johnstad (Geophysical Prospecting, 2005). "One-dimensional random patchy saturation model for velocity and attenuation in porous rocks" by Müller and Gurevich (Geophysics, 2004). "Velocity-saturation relation for partially saturated rocks with fractal pore fluid distribution" by Müller et al. (Geophysical Research Letters, 2008). "Seismic attenuation due to wave-induced flow" by Pride et al. (Journal of Geophysical Research, 2004). "Comparative review of theoretical models for elastic wave attenuation and dispersion in partially saturated rocks" by Toms et al. (Soil Dynamics and Earthquake Engineering, 2006). "Seismic attenuation in porous rocks with random patchy saturation" by Toms et al. (Geophysical Prospecting, 2007). "Acoustics of random patchy saturation" by Toms et al. (EAGE 2007 Extended Abstracts)."Modelng P-wave velocities from X-ray tomographic images of partially saturated porous rock" by Toms et al. (EAGE 2007 Extended Abstracts). "Computed seismic speeds and attenuation in rocks with partial gas saturation" by White (GeophYsICS, 1975). "Low frequency seismic waves in fluid-saturated layered rocks" by White et al. (Izvestija Academy of Sciences USSR, Physics of Solid Earth, 1975). TLE

Acknowledgments: The financial support of the Australian Research Council (project DP0771044), the Deutsche Forschungsgemeinschaft (contract MU1725/1-3), sponsors of the Curtin Reservoir Geophysics Consortium, and the Industry and Government Uptake Program Grant is gratefully acknowledged. Numerical simulations are carried out on iVEC, the hub of advanced computing in Western Australia.

Corresponding author: tobias.mueller@csiro.au 